\title{
Correction to: Quantifying the Cost of Distrust: Comparing Blockchain and Cloud Services for Business Process Execution
}

\author{
Paul Rimba $^{1} \cdot$ An Binh $\operatorname{Tran}^{1} \cdot$ Ingo Weber $^{1,2} \cdot$ Mark Staples $^{1,2} \cdot$ Alexander Ponomarev $^{1} \cdot \mathrm{Xiwei}^{\mathrm{X}} \mathrm{Xu}^{1,2}$ \\ Published online: 18 March 2019 \\ (C) Springer Science+Business Media, LLC, part of Springer Nature 2019
}

\section{Correction to: Information Systems Frontiers (2019) \\ https://doi.org/10.1007/s10796-018-9876-1}

The original version of this article, unfortunately, included the incorrect version of Fig. 1. We have attached a corrected version of Fig. 1 below. The model that was used for the testing and implementation is the right version. Therefore, the incorrect figure has not impacted the measurements and the conclusion of the article. The authors apologize for this error.

The online version of the original article can be found at https://doi.org/ 10.1007/s10796-018-9876-1

Paul Rimba

paul.rimba@data61.csiro.au

An Binh Tran

anbinh.tran@data61.csiro.au

Ingo Weber

ingo.weber@data61.csiro.au

Mark Staples

mark.staples@data61.csiro.au

Alexander Ponomarev

alex.ponomarev@data61.csiro.au

Xiwei Xu

xiwei.xu@data61.csiro.au

1 Data61, CSIRO, Sydney, Australia

2 School of Computer Science and Engineering, UNSW,

Sydney, Australia 


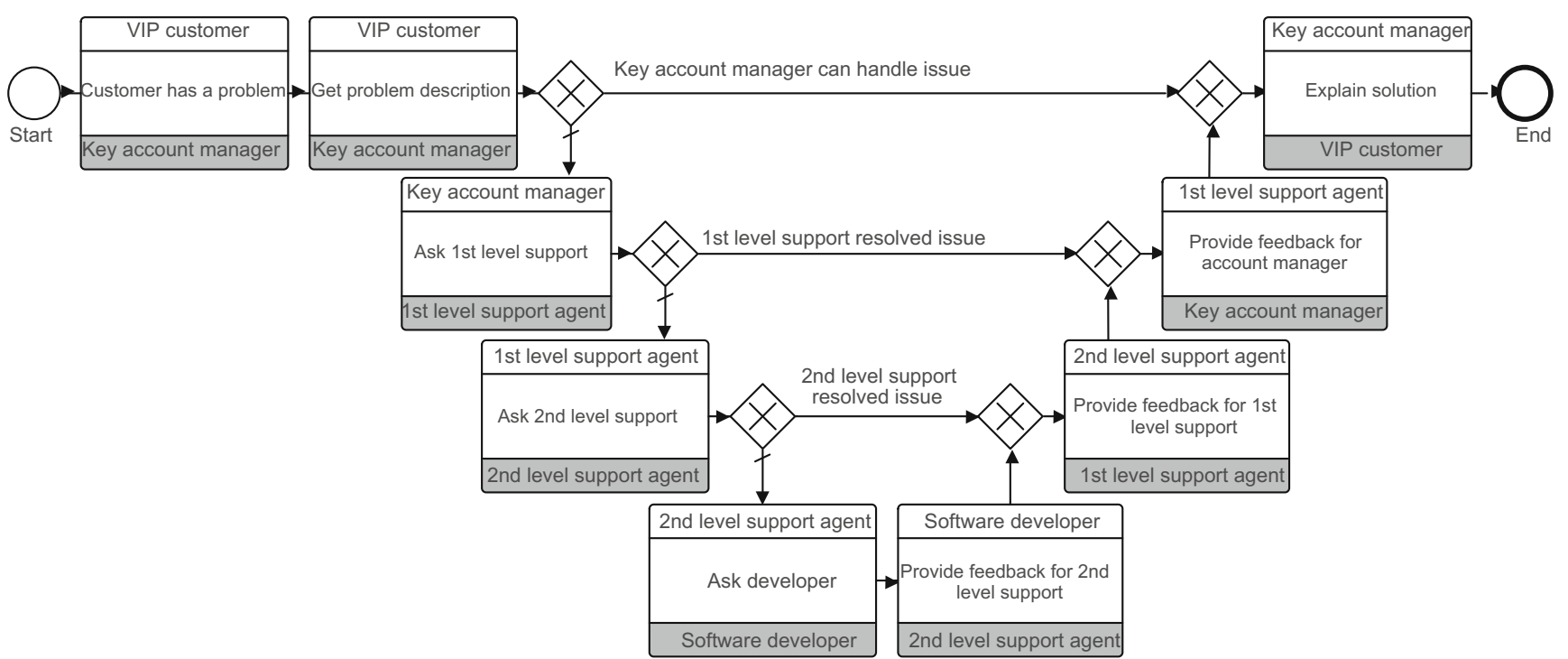

Publisher's Note Springer Nature remains neutral with regard to jurisdictional claims in published maps and institutional affiliations. 